\title{
Crystal Bases, Path Models, and a Twining Character Formula for Demazure Modules
}

\author{
By
}

\author{
Daisuke SAGAKI*
}

\begin{abstract}
We give a combinatorial proof of a twining character formula for Demazure modules, by combining the isomorphism theorem between path models and crystal bases with our previous result about Lakshmibai-Seshadri paths fixed by a diagram automorphism.
\end{abstract}

\section{§0. Introduction}

In [FRS] and [FSS], they introduced new character-like quantities corresponding to a graph automorphism of a Dynkin diagram, called twining characters, for certain Verma modules and integrable highest weight modules over a symmetrizable Kac-Moody algebra, and gave twining character formulas for them. Recently, the notion of twining characters has naturally been extended to various modules, and formulas for them has been given $([\mathrm{KN}],[\mathrm{KK}]$, [N1][N4]).

The purpose of this paper is to give a twining character formula for Demazure modules over a symmetrizable Kac-Moody algebra. Our formula is an extension of one of the main results in $[\mathrm{KN}]$, which describes the twining characters of Demazure modules over a finite-dimensional semi-simple Lie algebra. While their proof is an algebro-geometric one, we give a combinatorial proof by using the theories of path models and crystal bases.

Communicated by M. Kashiwara. Received May 14, 2001.

2001 Mathematics Subject Classification(s): Primary 17B67; Secondary 17B37, 20 G42.

Key words: crystal base, path model, twining character.

* Graduate School of Mathematics, University of Tsukuba, Tsukuba 305-8571, Japan.

e-mail: sagaki@math.tsukuba.ac.jp 
Let us explain our formula more precisely. Let $\mathfrak{g}=\mathfrak{g}(A)=\mathfrak{n}_{-} \oplus \mathfrak{h} \oplus \mathfrak{n}_{+}$ be a symmetrizable Kac-Moody algebra over $\mathbb{Q}$ associated to a generalized Cartan matrix $A=\left(a_{i j}\right)_{i, j \in I}$ of finite size, where $\mathfrak{h}$ is the Cartan subalgebra, $\mathfrak{n}_{+}$the sum of positive root spaces, and $\mathfrak{n}_{-}$the sum of negative root spaces, and let $\omega: I \rightarrow I$ be a (Dynkin) diagram automorphism, that is, a bijection $\omega: I \rightarrow I$ satisfying $a_{\omega(i), \omega(j)}=a_{i j}$ for all $i, j \in I$. It is known that a diagram automorphism induces a Lie algebra automorphism $\omega \in \operatorname{Aut}(\mathfrak{g})$ that preserves the triangular decomposition of $\mathfrak{g}$. Then we define a linear automorphism $\omega^{*} \in \operatorname{GL}\left(\mathfrak{h}^{*}\right)$ by $\left(\omega^{*}(\lambda)\right)(h):=\lambda(\omega(h))$ for $\lambda \in \mathfrak{h}^{*}, h \in \mathfrak{h}$. We set $\left(\mathfrak{h}^{*}\right)^{0}:=$ $\left\{\lambda \in \mathfrak{h}^{*} \mid \omega^{*}(\lambda)=\lambda\right\}$, and call its elements symmetric weights. We also set $\widetilde{W}:=\left\{w \in W \mid w \omega^{*}=\omega^{*} w\right\}$.

Further we define a "folded" matrix $\widehat{A}$ associated to $\omega$, which is again a symmetrizable GCM if $\omega$ satisfies a certain condition, called the linking condition (we assume it throughout this paper). Then the Kac-Moody algebra $\widehat{\mathfrak{g}}=\mathfrak{g}(\widehat{A})$ associated to $\widehat{A}$ is called the orbit Lie algebra. We denote by $\widehat{\mathfrak{h}}$ the Cartan subalgebra of $\widehat{\mathfrak{g}}$ and by $\widehat{W}$ the Weyl group of $\widehat{\mathfrak{g}}$. Then there exist a linear isomorphism $P_{\omega}^{*}: \widehat{\mathfrak{h}}^{*} \rightarrow\left(\mathfrak{h}^{*}\right)^{0}$ and a group isomorphism $\Theta: \widehat{W} \rightarrow \widetilde{W}$ such that $\Theta(\widehat{w})=P_{\omega}^{*} \circ \widehat{w} \circ\left(P_{\omega}^{*}\right)^{-1}$ for all $\widehat{w} \in \widehat{W}$.

Let $\lambda$ be a dominant integral weight. Denote by $L(\lambda)=\bigoplus_{\chi \in \mathfrak{h}^{*}} L(\lambda)_{\chi}$ the irreducible highest weight $\mathfrak{g}$-module of highest weight $\lambda$. Then, for $w \in W$, we define the Demazure module $L_{w}(\lambda)$ of lowest weight $w(\lambda)$ in $L(\lambda)$ by $L_{w}(\lambda):=$ $U(\mathfrak{b}) u_{w(\lambda)}$, where $u_{w(\lambda)} \in L(\lambda)_{w(\lambda)} \backslash\{0\}$ and $U(\mathfrak{b})$ is the universal enveloping algebra of the Borel subalgebra $\mathfrak{b}:=\mathfrak{h} \oplus \mathfrak{n}_{+}$of $\mathfrak{g}$. If $\lambda$ is symmetric, then we have a (unique) linear automorphism $\tau_{\omega}: L(\lambda) \rightarrow L(\lambda)$ such that

$$
\tau_{\omega}(x v)=\omega^{-1}(x) \tau_{\omega}(v) \quad \text { for all } x \in \mathfrak{g}, v \in L(\lambda)
$$

and $\tau_{\omega}\left(u_{\lambda}\right)=u_{\lambda}$ with $u_{\lambda}$ a (nonzero) highest weight vector of $L(\lambda)$. Then it is easily seen that the Demazure module $L_{w}(\lambda)$ with $w \in \widetilde{W}$ is $\tau_{\omega}$-stable. Here we define the twining character $\operatorname{ch}^{\omega}\left(L_{w}(\lambda)\right)$ of $L_{w}(\lambda)$ by:

$$
\operatorname{ch}^{\omega}\left(L_{w}(\lambda)\right):=\sum_{\chi \in\left(\mathfrak{h}^{*}\right)^{0}} \operatorname{tr}\left(\left.\tau_{\omega}\right|_{L_{w}(\lambda)_{\chi}}\right) e(\chi) .
$$

Our main theorem is the following:

Theorem. Let $\lambda$ be a symmetric dominant integral weight and $w \in \widetilde{W}$. Set $\widehat{\lambda}:=\left(P_{\omega}^{*}\right)^{-1}(\lambda)$ and $\widehat{w}:=\Theta^{-1}(w)$. Then we have

$$
\operatorname{ch}^{\omega}\left(L_{w}(\lambda)\right)=P_{\omega}^{*}\left(\operatorname{ch} \widehat{L}_{\widehat{w}}(\widehat{\lambda})\right),
$$

where $\widehat{L}_{\widehat{w}}(\widehat{\lambda})$ is the Demazure module of lowest weight $\widehat{w}(\widehat{\lambda})$ in the irreducible highest weight module $\widehat{L}(\widehat{\lambda})$ of highest weight $\widehat{\lambda}$ over the orbit Lie algebra $\widehat{\mathfrak{g}}$. 
The starting point of this work is the main result in [NS1]. Denote by $\mathbb{B}(\lambda)$ the set of Lakshmibai-Seshadri paths (L-S paths for short) of class $\lambda$, where the L-S paths of class $\lambda$ are, by definition, piecewise linear, continuous maps $\pi$ : $[0,1] \rightarrow \mathfrak{h}^{*}$ parametrized by sequences of elements in $W \lambda$ and rational numbers with a certain condition, called the chain condition. In [Li1], Littelmann showed that there exists a subset $\mathbb{B}_{w}(\lambda)$ of $\mathbb{B}(\lambda)$ such that

$$
\sum_{\pi \in \mathbb{B}_{w}(\lambda)} e(\pi(1))=\operatorname{ch} L_{w}(\lambda) .
$$

For $\pi \in \mathbb{B}(\lambda)$, we define $\omega^{*}(\pi):[0,1] \rightarrow \mathfrak{h}^{*}$ by $\left(\omega^{*}(\pi)\right)(t):=\omega^{*}(\pi(t))$. If $\lambda$ is symmetric and $w \in \widetilde{W}$, then $\mathbb{B}_{w}(\lambda)$ is $\omega^{*}$-stable. We denote by $\mathbb{B}_{w}^{0}(\lambda)$ the set of elements of $\mathbb{B}_{w}(\lambda)$ fixed by $\omega^{*}$. We see from the main result of [NS1] that

$$
\sum_{\pi \in \mathbb{B}_{w}^{0}(\lambda)} e(\pi(1))=P_{\omega}^{*}\left(\operatorname{ch} \widehat{L}_{\widehat{w}}(\widehat{\lambda})\right) .
$$

In this paper, we prove that the left-hand side is, in fact, equal to $\operatorname{ch}^{\omega}\left(L_{w}(\lambda)\right)$.

In order to prove the equality $\operatorname{ch}^{\omega}\left(L_{w}(\lambda)\right)=\sum_{\pi \in \mathbb{B}_{w}^{0}(\lambda)} e(\pi(1))$, we introduce a "quantum version" of twining characters, called $q$-twining characters. Let $U_{q}(\mathfrak{g})$ be the quantum group associated to the Kac-Moody algebra $\mathfrak{g}$ over the field $\mathbb{Q}(q)$ of rational functions in $q$, and $V(\lambda)=\bigoplus_{\chi \in \mathfrak{h}^{*}} V(\lambda)_{\chi}$ the irreducible highest weight $U_{q}(\mathfrak{g})$-module of highest weight $\lambda$. For $w \in W$, the quantum Demazure module $V_{w}(\lambda)$ is defined by

$$
V_{w}(\lambda):=U_{q}^{+}(\mathfrak{g}) u_{w(\lambda)},
$$

where $u_{w(\lambda)} \in V(\lambda)_{w(\lambda)} \backslash\{0\}$, and $U_{q}^{+}(\mathfrak{g})$ is the "positive part" of $U_{q}(\mathfrak{g})$. A diagram automorphism $\omega$ induces a $\mathbb{Q}(q)$-algebra automorphism $\omega_{q}$ of $U_{q}(\mathfrak{g})$. Assume that $\lambda$ is symmetric. Then we get a $\mathbb{Q}(q)$-linear automorphism $\tau_{\omega_{q}}$ of $V(\lambda)$ that has the same properties as $\tau_{\omega}$ in the Lie algebra case. Note that $V_{w}(\lambda)$ is stable under $\tau_{\omega_{q}}$ if $w \in \widetilde{W}$. Then we define the $q$-twining character $\operatorname{ch}_{q}^{\omega}\left(V_{w}(\lambda)\right)$ of $V_{w}(\lambda)$ by

$$
\operatorname{ch}_{q}^{\omega}\left(V_{w}(\lambda)\right):=\sum_{\chi \in\left(\mathfrak{h}^{*}\right)^{0}} \operatorname{tr}\left(\left.\tau_{\omega_{q}}\right|_{V_{w}(\lambda)_{\chi}}\right) e(\chi)
$$

where the traces are naively elements of $\mathbb{Q}(q)$ (in fact, they are elements of $\left.\mathbb{Q}\left[q, q^{-1}\right]\right)$. We show that the specialization of the $q$-twining character above by $q=1$ is equal to the (ordinary) twining character $\operatorname{ch}^{\omega}\left(L_{w}(\lambda)\right)$, that is,

$$
\left.\operatorname{ch}_{q}^{\omega}\left(V_{w}(\lambda)\right)\right|_{q=1}=\operatorname{ch}^{\omega}\left(L_{w}(\lambda)\right)
$$


The advantage of considering a quantum version is the existence of a basis of $V_{w}(\lambda)$ compatible with $\tau_{\omega_{q}}$. Let $(\mathcal{L}(\lambda), \mathcal{B}(\lambda))$ be the (lower) crystal base of $V(\lambda)$. In [Kas3], Kashiwara showed that, for each $w \in W$, there exists a subset $\mathcal{B}_{w}(\lambda)$, called the Demazure crystal for $V_{w}(\lambda)$, of $\mathcal{B}(\lambda)$ such that

$$
V_{w}(\lambda):=\bigoplus_{b \in \mathcal{B}_{w}(\lambda)} \mathbb{Q}(q) G_{\lambda}(b)
$$

where $G_{\lambda}(b)$ denotes the (lower) global base introduced in [Kas2]. We prove that $\tau_{\omega_{q}}$ stabilizes the basis $\left\{G_{\lambda}(b) \mid b \in \mathcal{B}_{w}(\lambda)\right\}$ of $V_{w}(\lambda)$.

By combining these facts and the equivalence theorem between path models $\mathbb{B}(\lambda)$ and crystal bases $\mathcal{B}(\lambda)$, which was proved by Kashiwara [Kas5] et al., we can obtain the desired equality above, and hence the our main theorem.

This paper is organized as follows. In Section 1 we review some facts about Kac-Moody algebras, diagram automorphisms, orbit Lie algebras, quantum groups, crystal bases, and path models. There we also define an algebra automorphism of the quantum group $U_{q}(\mathfrak{g})$ induced from a diagram automorphism. In Section 2, we recall the definitions of the twining characters of $L(\lambda)$ and $L_{w}(\lambda)$, and then introduce the $q$-twining characters of the irreducible highest weight $U_{q}(\mathfrak{g})$-module $V(\lambda)$ and the quantum Demazure module $V_{w}(\lambda)$. Furthermore, we explain that the $q$-twining characters of $V(\lambda)$ and $V_{w}(\lambda)$ are $q$ analogues of the twining characters of $L(\lambda)$ and $L_{w}(\lambda)$, respectively. In Section 3 we give a proof of our main theorem by calculating the $q$-twining character of $V_{w}(\lambda)$.

\section{$\S 1$. Preliminaries}

\section{§1.1. Kac-Moody algebras and diagram automorphisms}

In this subsection, we review some basic facts about Kac-Moody algebras from [Kac] and [MP], and about diagram automorphisms from [FRS] and [FSS].

Let $A=\left(a_{i j}\right)_{i, j \in I}$ be a symmetrizable generalized Cartan matrix (GCM for short) indexed by a finite set $I$. Then there exists a diagonal matrix $D=$ $\operatorname{diag}\left(\varepsilon_{i}\right)_{i \in I}$ with $\varepsilon_{i} \in \mathbb{Q}_{>0}$ such that $D^{-1} A$ is a symmetric matrix. Let $\omega: I \rightarrow$ $I$ be a diagram automorphism of order $N$, that is, a bijection $\omega: I \rightarrow I$ of order $N$ such that $a_{\omega(i), \omega(j)}=a_{i j}$ for all $i, j \in I$.

Remark 1. Set

$$
D^{\prime}=\operatorname{diag}\left(\varepsilon_{i}^{\prime}\right)_{i \in I}:=\operatorname{diag}\left(\frac{1}{\sum_{k=0}^{N-1} \varepsilon_{\omega^{k}(i)}^{-1}}\right)_{i \in I} .
$$


Then we see that $\varepsilon_{\omega(i)}^{\prime}=\varepsilon_{i}^{\prime}$ and $\left(D^{\prime}\right)^{-1} A$ is a symmetric matrix. Hence, by replacing $D$ with $D^{\prime}$ above if necessary, we may (and will henceforth) assume that $\varepsilon_{\omega(i)}=\varepsilon_{i}($ see also [N1, Section 3.1]).

We take a realization $\left(\mathfrak{h}, \Pi, \Pi^{\vee}\right)$ of the GCM $A=\left(a_{i j}\right)_{i, j \in I}$ over $\mathbb{Q}$ and linear automorphisms $\omega: \mathfrak{h} \rightarrow \mathfrak{h}$ and $\omega^{*}: \mathfrak{h}^{*} \rightarrow \mathfrak{h}^{*}$ as follows (cf. [Kac, Exercises 1.15 and 1.16]). Let $\mathfrak{h}^{\prime}$ be an $n$-dimensional vector space over $\mathbb{Q}$ with $\Pi^{\vee}:=\left\{\alpha_{i}^{\vee}\right\}_{i \in I}$ a basis. We define a $\mathbb{Q}$-linear automorphism $\omega^{\prime}: \mathfrak{h}^{\prime} \rightarrow \mathfrak{h}^{\prime}$ by $\omega^{\prime}\left(\alpha_{i}^{\vee}\right)=\alpha_{\omega(i)}^{\vee}$, and $\omega^{\prime \prime}:\left(\mathfrak{h}^{\prime}\right)^{*} \rightarrow\left(\mathfrak{h}^{\prime}\right)^{*}$ by $\left(\omega^{\prime \prime}(\lambda)\right)(h):=\lambda\left(\left(\omega^{\prime}\right)^{-1}(h)\right)$ for $\lambda \in\left(\mathfrak{h}^{\prime}\right)^{*}$ and $h \in \mathfrak{h}^{\prime}$. We also define $\varphi: \mathfrak{h}^{\prime} \rightarrow\left(\mathfrak{h}^{\prime}\right)^{*}$ by $\left(\varphi\left(\alpha_{i}^{\vee}\right)\right)\left(\alpha_{j}^{\vee}\right)=a_{i j}$. It can be readily seen that $\omega^{\prime \prime} \circ \varphi=\varphi \circ \omega^{\prime}$. This means that $\operatorname{Im} \varphi$ is $\omega^{\prime \prime}$-stable, and hence we can take a complementary subspace $\mathfrak{h}^{\prime \prime}$ of $\operatorname{Im} \varphi$ in $\left(\mathfrak{h}^{\prime}\right)^{*}$ that is also $\omega^{\prime \prime}$-stable. Now set $\mathfrak{h}:=\mathfrak{h}^{\prime} \oplus \mathfrak{h}^{\prime \prime}$, and $\Pi:=\left\{\alpha_{i}\right\}_{i \in I}$, where $\alpha_{i} \in \mathfrak{h}^{*}$ is defined by

$$
\alpha_{i}\left(\sum_{j \in I} c_{j} \alpha_{j}^{\vee}+h^{\prime \prime}\right):=\sum_{j \in I} c_{j}\left(\varphi\left(\alpha_{j}^{\vee}\right)\right)\left(\alpha_{i}^{\vee}\right)+h^{\prime \prime}\left(\alpha_{i}^{\vee}\right) \quad \text { for } \quad h^{\prime \prime} \in \mathfrak{h}^{\prime \prime} .
$$

Then we see that $\Pi$ is a linearly independent subset of $\mathfrak{h}^{*}$. Furthermore, since $\operatorname{dim}_{\mathbb{Q}} \mathfrak{h}^{\prime \prime}=\# I-\operatorname{dim}_{\mathbb{Q}} \operatorname{Im} \varphi=\# I-\operatorname{rank} A$, we have $\operatorname{dim}_{\mathbb{Q}} \mathfrak{h}=2 \# I-\operatorname{rank} A$ Hence $\left(\mathfrak{h}, \Pi, \Pi^{\vee}\right)$ is a (minimal) realization of the GCM $A$. We define a $\mathbb{Q}$-linear automorphism $\omega: \mathfrak{h} \rightarrow \mathfrak{h}$ by $\omega\left(h^{\prime}+h^{\prime \prime}\right):=\omega^{\prime}\left(h^{\prime}\right)+\omega^{\prime \prime}\left(h^{\prime \prime}\right)$ for $h^{\prime} \in \mathfrak{h}^{\prime}$ and $h^{\prime \prime} \in \mathfrak{h}^{\prime \prime}$, and the transposed map $\omega^{*}: \mathfrak{h}^{*} \rightarrow \mathfrak{h}^{*}$ by $\left(\omega^{*}(\lambda)\right)(h)=\lambda(\omega(h))$ for $\lambda \in \mathfrak{h}^{*}$ and $h \in \mathfrak{h}$. Then we can check, by using (1.1), that $\omega^{*}\left(\alpha_{i}\right)=\alpha_{\omega^{-1}(i)}$ for each $i \in I$.

Here, as in [Kac, Section 2.1], we define the (standard) nondegenerate symmetric bilinear form $(\cdot, \cdot)$ on $\mathfrak{h}$ associated to the decomposition $\mathfrak{h}=\mathfrak{h}^{\prime} \oplus \mathfrak{h}^{\prime \prime}$ above. We set

$$
\begin{cases}\left(\alpha_{i}^{\vee}, h\right):=\alpha_{i}(h) \varepsilon_{i} & \text { for } i \in I, h \in \mathfrak{h}, \\ \left(h, h^{\prime}\right):=0 & \text { for } h, h^{\prime} \in \mathfrak{h}^{\prime \prime}\end{cases}
$$

Then it follows from the construction above and Remark 1 that $\left(\omega(h), \omega\left(h^{\prime}\right)\right)=$ $\left(h, h^{\prime}\right)$ for all $h, h^{\prime} \in \mathfrak{h}$. We denote also by $(\cdot, \cdot)$ the nondegenerate symmetric bilinear form on $\mathfrak{h}^{*}$ induced from the bilinear form on $\mathfrak{h}$. Then $\left(\omega^{*}(\lambda), \omega^{*}\left(\lambda^{\prime}\right)\right)=$ $\left(\lambda, \lambda^{\prime}\right)$ for all $\lambda, \lambda^{\prime} \in \mathfrak{h}^{*}$. We set

$$
\left(\mathfrak{h}^{*}\right)^{0}:=\left\{\lambda \in \mathfrak{h}^{*} \mid \omega^{*}(\lambda)=\lambda\right\}, \quad \mathfrak{h}^{0}:=\{h \in \mathfrak{h} \mid \omega(h)=h\} .
$$

Elements of $\left(\mathfrak{h}^{*}\right)^{0}$ are called symmetric weights. Note that $\left(\mathfrak{h}^{*}\right)^{0}$ can be identified with $\left(\mathfrak{h}^{0}\right)^{*}$ in a natural way. 
Remark 2. Let $\rho$ be a Weyl vector, i.e., an element of $\mathfrak{h}^{*}$ such that $\rho\left(\alpha_{i}^{\vee}\right)=1$ for all $i \in I$. Then, by replacing $\rho$ with $(1 / N) \sum_{k=0}^{N-1}\left(\omega^{*}\right)^{k}(\rho)$ if necessary, we may (and will henceforth) assume that a Weyl vector $\rho$ is a symmetric weight.

Let $\mathfrak{g}=\mathfrak{g}(A)$ be the Kac-Moody algebra over $\mathbb{Q}$ associated to the GCM $A$ with $\mathfrak{h}$ the Cartan subalgebra, $\Pi=\left\{\alpha_{i}\right\}_{i \in I}$ the set of simple roots, and $\Pi^{\vee}=$ $\left\{\alpha_{i}^{\vee}\right\}_{i \in I}$ the set of simple coroots. Denote by $\left\{x_{i}, y_{i} \mid i \in I\right\}$ the Chevalley generators, where $x_{i}$ (resp. $y_{i}$ ) spans the root space of $\mathfrak{g}$ corresponding to $\alpha_{i}$ (resp. $-\alpha_{i}$ ). The Weyl group $W$ of $\mathfrak{g}$ is defined by $W:=\left\langle r_{i} \mid i \in I\right\rangle$, where $r_{i}$ is the simple reflection with respect to $\alpha_{i}$. The following lemma is obvious from the definitions of Kac-Moody algebras and the linear map $\omega: \mathfrak{h} \rightarrow \mathfrak{h}$ above.

Lemma 1.1. The $\mathbb{Q}$-linear map $\omega: \mathfrak{h} \rightarrow \mathfrak{h}$ above can be extended to a Lie algebra automorphism $\omega \in \operatorname{Aut}(\mathfrak{g})$ of order $N$ such that $\omega\left(x_{i}\right)=x_{\omega(i)}$ and $\omega\left(y_{i}\right)=y_{\omega(i)}$.

Let $\lambda$ be a dominant integral weight. Denote by $L(\lambda)=\bigoplus_{\chi \in \mathfrak{h}^{*}} L(\lambda)_{\chi}$ the irreducible highest weight $\mathfrak{g}$-module of highest weight $\lambda$, where $L(\lambda)_{\chi}$ is the $\chi$-weight space of $L(\lambda)$. We set $\mathfrak{b}:=\mathfrak{h} \oplus \mathfrak{n}_{+}$, where $\mathfrak{n}_{+}$is the sum of positive root spaces of $\mathfrak{g}$. For $w \in W$, the Demazure module $L_{w}(\lambda) \subset L(\lambda)$ of lowest weight $w(\lambda)$ is defined by $L_{w}(\lambda):=U(\mathfrak{b}) u_{w(\lambda)}$, where $U(\mathfrak{b})$ is the universal enveloping algebra of $\mathfrak{b}$ and $u_{w(\lambda)} \in L(\lambda)_{w(\lambda)} \backslash\{0\}$. In addition, for each $i \in I$, we define the Demazure operator $D_{i}$ by

$$
D_{i}(e(\lambda)):=\frac{e(\lambda+\rho)-e\left(r_{i}(\lambda+\rho)\right)}{1-e\left(-\alpha_{i}\right)} e(-\rho) \quad \text { for } \quad \lambda \in \mathfrak{h}^{*} .
$$

By $[\mathrm{Kas} 3],[\mathrm{Ku}]$ and $[\mathrm{M}]$, we know the following character formula for Demazure modules.

Theorem 1.1. $\quad$ Let $\lambda$ be a dominant integral weight and $w \in W$. Assume that $w=r_{i_{1}} r_{i_{2}} \cdots r_{i_{k}}$ is a reduced expression of $w$. Then we have

$$
\operatorname{ch} L_{w}(\lambda)=D_{i_{1}} \circ D_{i_{2}} \circ \cdots \circ D_{i_{k}}(e(\lambda)) .
$$

Remark 3. The Demazure operators $\left\{D_{i}\right\}_{i \in I}$ satisfy the braid relations (see $[D]$ ). Hence the right-hand side of (1.4) above does not depend on the choice of a reduced expression of $w$. 


\section{§1.2. Orbit Lie algebras}

In this subsection, we review the notion of orbit Lie algebras. For details, see $[$ FRS] and [FSS].

We set

$$
c_{i j}:=\sum_{k=0}^{N_{j}-1} a_{i, \omega^{k}(j)} \quad \text { for } \quad i, j \in I \quad \text { and } \quad c_{i}:=c_{i i} \text { for } i \in I,
$$

where $N_{i}$ is the number of elements of the $\omega$-orbit of $i \in I$ in $I$. From now on, we assume that a diagram automorphism $\omega$ satisfies

$$
c_{i}=1 \text { or } 2 \text { for each } i \in I .
$$

This condition is called the linking condition. Here we choose a complete set $\widehat{I}$ of representatives of the $\omega$-orbits in $I$, and define a matrix $\widehat{A}=\left(\widehat{a}_{i j}\right)_{i, j \in \widehat{I}}$ by

$$
\widehat{A}=\left(\widehat{a}_{i j}\right)_{i, j \in \widehat{I}}:=\left(2 c_{i j} / c_{j}\right)_{i, j \in \widehat{I}} .
$$

Proposition 1.1 ([FSS, Section 2.2]). The matrix $\widehat{A}=\left(\widehat{a}_{i j}\right)_{i, j \in \hat{I}}$ is a symmetrizable GCM.

The Kac-Moody algebra $\widehat{\mathfrak{g}}:=\mathfrak{g}(\widehat{A})$ over $\mathbb{Q}$ associated to the GCM $\widehat{A}$ is called the orbit Lie algebra (associated to the diagram automorphism $\omega$ ). Denote by $\widehat{\mathfrak{h}}$ the Cartan subalgebra of $\widehat{\mathfrak{g}}$, and by $\widehat{\Pi}=\left\{\widehat{\alpha}_{i}\right\}_{i \in \widehat{I}}$ and $\widehat{\Pi}^{\vee}=\left\{\widehat{\alpha}_{i}^{\vee}\right\}_{i \in \widehat{I}}$ the set of simple roots and simple coroots of $\widehat{\mathfrak{g}}$, respectively.

As in [FRS, Section 2], we have a $\mathbb{Q}$-linear isomorphism $P_{\omega}: \mathfrak{h}^{0} \rightarrow \widehat{\mathfrak{h}}$ such that

$$
\begin{cases}P_{\omega}\left(\frac{1}{N_{i}} \sum_{k=0}^{N_{i}-1} \alpha_{\omega^{k}(i)}^{\vee}\right)=\widehat{\alpha}_{i}^{\vee} & \text { for each } i \in \widehat{I}, \\ \left(P_{\omega}(h), P_{\omega}\left(h^{\prime}\right)\right)=\left(h, h^{\prime}\right) & \text { for all } h, h^{\prime} \in \mathfrak{h}^{0},\end{cases}
$$

where we denote also by $(\cdot, \cdot)$ the (standard) nondegenerate symmetric bilinear form on $\widehat{\mathfrak{h}}$. Let $P_{\omega}^{*}: \widehat{\mathfrak{h}}^{*} \rightarrow\left(\mathfrak{h}^{0}\right)^{*} \cong\left(\mathfrak{h}^{*}\right)^{0}$ be the transposed map of $P_{\omega}$ defined by

$$
\left(P_{\omega}^{*}(\widehat{\lambda})\right)(h):=\widehat{\lambda}\left(P_{\omega}(h)\right) \quad \text { for } \widehat{\lambda} \in \widehat{\mathfrak{h}}^{*}, h \in \mathfrak{h}^{0}
$$

Proposition 1.2 ([FRS, Proposition 3.3]). Set $\widetilde{W}:=\left\{w \in W \mid w w^{*}=\right.$ $\left.\omega^{*} w\right\}$. Then there exists a group isomorphism $\Theta: \widehat{W} \rightarrow \widetilde{W}$ such that $\Theta(\widehat{w})=$ 
$P_{\omega}^{*} \circ \widehat{w} \circ\left(P_{\omega}^{*}\right)^{-1}$ for each $\widehat{w} \in \widehat{W}$.

\section{$\S 1.3$. Quantum groups}

From now on, we take the bilinear form $(\cdot, \cdot)$ in such a way that $\left(\alpha_{i}, \alpha_{i}\right) \in$ $\mathbb{Z}_{>0}$ for all $i \in I$. Let $P \subset \mathfrak{h}^{*}$ be an $\omega^{*}$-stable integral weight lattice such that $\alpha_{i} \in P$ for all $i \in I$, and set $P_{+}:=\left\{\lambda \in P \mid \lambda\left(\alpha_{i}^{\vee}\right) \in \mathbb{Z}_{\geq 0}\right.$ for all $\left.i \in I\right\}$. Notice that the dual lattice $P^{\vee}:=\operatorname{Hom}_{\mathbb{Z}}(P, \mathbb{Z})$ of $P$ is stable under $\omega$. The quantum group (or quantized universal enveloping algebra) $U_{q}(\mathfrak{g})$ associated to $\mathfrak{g}$ is, by definition, the algebra generated by the symbols $X_{i}, Y_{i}$ and $q^{h}\left(h \in P^{\vee}\right)$ over the field $\mathbb{Q}(q)$ of rational functions in $q$ with the following defining relations:

$$
\left\{\begin{array}{l}
q^{0}=1, \quad q^{h_{1}} q^{h_{2}}=q^{h_{1}+h_{2}} \quad \text { for } h_{1}, h_{2} \in P^{\vee}, \\
q^{h} X_{i} q^{-h}=q^{\alpha_{i}(h)} X_{i}, \quad q^{h} Y_{i} q^{-h}=q^{-\alpha_{i}(h)} Y_{i} \quad \text { for } \quad i \in I, h \in P^{\vee}, \\
{\left[X_{i}, Y_{i}\right]=\delta_{i j} \frac{t_{i}-t_{i}^{-1}}{q_{i}-q_{i}^{-1}} \quad \text { for } i \in I,} \\
\sum_{k=0}^{1-a_{i j}}(-1)^{k} X_{i}^{(k)} X_{j} X_{i}^{\left(1-a_{i j}-k\right)}=0 \quad \text { for } i, j \in I \text { with } i \neq j, \\
\sum_{k=0}^{1-a_{i j}}(-1)^{k} Y_{i}^{(k)} Y_{j} Y_{i}^{\left(1-a_{i j}-k\right)}=0 \quad \text { for } i, j \in I \text { with } i \neq j .
\end{array}\right.
$$

Here we have used the following notation:

$$
\begin{gathered}
q_{i}:=q^{\left(\alpha_{i}, \alpha_{i}\right)}, \quad t_{i}:=q^{\left(\alpha_{i}, \alpha_{i}\right) \alpha_{i}^{\vee}} \\
{[n]_{i}:=\frac{q_{i}^{n}-q_{i}^{-n}}{q_{i}-q_{i}^{-1}}, \quad[n]_{i} !:=\prod_{k=1}^{n}[k]_{i}, \quad \text { and } \quad X_{i}^{(n)}:=\frac{X_{i}^{n}}{[n]_{i} !}, \quad Y_{i}^{(n)}:=\frac{Y_{i}^{n}}{[n]_{i} !} .}
\end{gathered}
$$

Lemma 1.2. $\quad$ There exists a unique $\mathbb{Q}(q)$-algebra automorphism $\omega_{q}$ of $U_{q}(\mathfrak{g})$ such that $\omega_{q}\left(X_{i}\right)=X_{\omega(i)}, \omega_{q}\left(Y_{i}\right)=Y_{\omega(i)}$, and $\omega_{q}\left(q^{h}\right)=q^{\omega(h)}$.

Proof. We need only show that the images of the generators by $\omega_{q}$ also satisfy the defining relations (1.9). However it can easily be checked by using the equalities $q_{\omega(i)}=q_{i},[n]_{\omega(i)}=[n]_{i}$, and $t_{\omega(i)}=t_{i}$.

Let $\lambda \in P_{+}$. Denote by $V(\lambda)=\bigoplus_{\chi \in \mathfrak{h}^{*}} V(\lambda)_{\chi}$ the irreducible highest weight $U_{q}(\mathfrak{g})$-module of highest weight $\lambda$, where $V(\lambda)_{\chi}$ is the $\chi$-weight space of $V(\lambda)$. It is known (cf. [Kas1, (1.2.7)]) that

$$
V(\lambda) \cong U_{q}^{-}(\mathfrak{g}) /\left(\sum_{i \in I} U_{q}^{-}(\mathfrak{g}) Y_{i}^{1+\lambda\left(\alpha_{i}^{\vee}\right)}\right)
$$


where $U_{q}^{-}(\mathfrak{g})$ is the $\mathbb{Q}(q)$-subalgebra of $U_{q}(\mathfrak{g})$ generated by $\left\{Y_{i}\right\}_{i \in I}$. For each $w \in W$, we define the quantum Demazure module $V_{w}(\lambda) \subset V(\lambda)$ of lowest weight $w(\lambda)$ by $V_{w}(\lambda):=U_{q}^{+}(\mathfrak{g}) u_{w(\lambda)}$, where $U_{q}^{+}(\mathfrak{g})$ is the $\mathbb{Q}(q)$-subalgebra of $U_{q}(\mathfrak{g})$ generated by $\left\{X_{i}\right\}_{i \in I}$, and where $u_{w(\lambda)} \in V(\lambda)_{w(\lambda)} \backslash\{0\}$.

\section{$\S 1.4$. Crystal bases and global bases}

In this subsection, we recall the definitions of (lower) crystal bases and (lower) global bases. For details, see [Ja] and [Kas1]-[Kas3].

First let us recall the definition of the Kashiwara operators $E_{i}, F_{i}$ on $V(\lambda)$. It is known that each element $u \in V(\lambda)_{\chi}$ can be uniquely written as $u=\sum_{k \geq 0} Y_{i}^{(k)} u_{k}$, where $u_{k} \in\left(\operatorname{ker} X_{i}\right) \cap V(\lambda)_{\chi+k \alpha_{i}}$. We define the $\mathbb{Q}(q)$-linear operators $E_{i}, F_{i}$ on $V(\lambda)$ by

$$
E_{i} u:=\sum_{k \geq 0} Y_{i}^{(k-1)} u_{k}, \quad F_{i} u:=\sum_{k \geq 0} Y_{i}^{(k+1)} u_{k} .
$$

Denote by $A_{0}$ the subring of $\mathbb{Q}(q)$ consisting of the rational functions in $q$ regular at $q=0$, and by $\mathcal{L}_{0}(\lambda)$ the $A_{0}$-submodule of $V(\lambda)$ generated by all elements of the form $F_{i_{1}} F_{i_{2}} \cdots F_{i_{k}} u_{\lambda}$, where $u_{\lambda}$ is a (nonzero) highest weight vector of $V(\lambda)$. Let $\mathcal{B}(\lambda) \subset \mathcal{L}_{0}(\lambda) / q \mathcal{L}_{0}(\lambda)$ be the set of nonzero images of $F_{i_{1}} F_{i_{2}} \cdots F_{i_{k}} u_{\lambda}$ by the canonical map ${ }^{-}: \mathcal{L}_{0}(\lambda) \rightarrow \mathcal{L}_{0}(\lambda) / q \mathcal{L}_{0}(\lambda)$. Then it is known from [Kas1, Theorem 2] that $\left(\mathcal{L}_{0}(\lambda), \mathcal{B}(\lambda)\right)$ is a (lower) crystal base of $V(\lambda)$, i.e.,

(1) $V(\lambda)=\mathbb{Q}(q) \otimes_{A_{0}} \mathcal{L}_{0}(\lambda)$,

(2) $\mathcal{L}_{0}(\lambda)=\bigoplus_{\chi \in \mathfrak{h}^{*}} \mathcal{L}_{0}(\lambda)_{\chi}$, where $\mathcal{L}_{0}(\lambda)_{\chi}=\mathcal{L}_{0}(\lambda) \cap V(\lambda)_{\chi}$,

(3) $E_{i} \mathcal{L}_{0}(\lambda) \subset \mathcal{L}_{0}(\lambda)$ and $F_{i} \mathcal{L}_{0}(\lambda) \subset \mathcal{L}_{0}(\lambda)$,

(4) $\mathcal{B}(\lambda)$ is a basis of the $\mathbb{Q}$-vector space $\mathcal{L}_{0}(\lambda) / q \mathcal{L}_{0}(\lambda)$,

(5) $E_{i} \mathcal{B}(\lambda) \subset \mathcal{B}(\lambda) \cup\{0\}$ and $F_{i} \mathcal{B}(\lambda) \subset \mathcal{B}(\lambda) \cup\{0\}$,

(6) $\mathcal{B}(\lambda)=\bigcup_{\chi \in \mathfrak{h} *} \mathcal{B}(\lambda)_{\chi}$ (disjoint union), where $\mathcal{B}(\lambda)_{\chi}:=\mathcal{B}(\lambda) \cap\left(\mathcal{L}_{0}(\lambda)_{\chi} / q \mathcal{L}_{0}(\lambda)_{\chi}\right)$,

(7) For $b_{1}, b_{2} \in \mathcal{B}(\lambda), b_{1}=F_{i} b_{2}$ if and only if $b_{2}=E_{i} b_{1}$.

Note that, by (3), we have the operators on $\mathcal{L}_{0}(\lambda) / q \mathcal{L}_{0}(\lambda)$ induced from $E_{i}, F_{i}$, which are also denoted by $E_{i}, F_{i}$ (cf. (5), (7)). 
Next we recall the notion of (lower) global bases. Set $V_{\mathbb{Q}}(\lambda):=U_{q}^{\mathbb{Q}}(\mathfrak{g}) u_{\lambda} \subset$ $V(\lambda)$, where $U_{q}^{\mathbb{Q}}(\mathfrak{g})$ is the $\mathbb{Q}\left[q, q^{-1}\right]$-subalgebra of $U_{q}(\mathfrak{g})$ generated by all $X_{i}^{(n)}$, $Y_{i}^{(n)}, q^{h}$, and

$$
\left\{\begin{array}{c}
q^{h} \\
n
\end{array}\right\}:=\prod_{k=1}^{n} \frac{q^{1-k} q^{h}-q^{k-1} q^{-h}}{q^{k}-q^{-k}}
$$

for $i \in I, n \in \mathbb{Z}_{\geq 0}, h \in P^{\vee}$. We define a $\mathbb{Q}$-algebra automorphism $\psi: U_{q}(\mathfrak{g}) \rightarrow$ $U_{q}(\mathfrak{g})$ by

$$
\begin{cases}\psi\left(X_{i}\right):=X_{i}, \psi\left(Y_{i}\right):=Y_{i} & \text { for } \quad i \in I, \\ \psi(q):=q^{-1}, \psi\left(q^{h}\right):=q^{-h} & \text { for } \quad h \in P^{\vee} .\end{cases}
$$

By virtue of (1.10), we can define the automorphism $\psi$ of $V(\lambda)$ by $\psi\left(x u_{\lambda}\right):=$ $\psi(x) u_{\lambda}$ for $x \in U_{q}^{-}(\mathfrak{g})$. Let $\mathcal{L}_{\infty}(\lambda)$ be the image of $\mathcal{L}_{0}(\lambda)$ by $\psi$. It is known from [Kas1] that the restriction of the canonical map ${ }^{-}$to $E(\lambda):=V_{\mathbb{Q}}(\lambda) \cap \mathcal{L}_{0}(\lambda) \cap$ $\mathcal{L}_{\infty}(\lambda)$ is an isomorphism from $E(\lambda)$ to $\mathcal{L}_{0}(\lambda) / q \mathcal{L}_{0}(\lambda)$ as $\mathbb{Q}$-vector spaces. We denote by $G_{\lambda}$ the inverse of this isomorphism. Then we have

$$
V(\lambda)=\bigoplus_{b \in \mathcal{B}(\lambda)} \mathbb{Q}(q) G_{\lambda}(b) .
$$

Moreover we have the following.

Theorem 1.2 ([Kas3, Proposition 3.2.3]). Let $\lambda \in P_{+}$and $w \in W$. Then there exists a subset $\mathcal{B}_{w}(\lambda)$ of $\mathcal{B}(\lambda)$ such that

$$
V_{w}(\lambda)=\bigoplus_{b \in \mathcal{B}_{w}(\lambda)} \mathbb{Q}(q) G_{\lambda}(b)
$$

\section{§1.5. Path models}

Let $\lambda \in P_{+}$. For $\mu, \nu \in W \lambda$, we write $\mu \geq \nu$ if there exist a sequence $\mu=\lambda_{0}, \lambda_{1}, \ldots, \lambda_{s}=\nu$ of elements in $W \lambda$ and a sequence $\beta_{1}, \ldots, \beta_{s}$ of positive real roots such that $\lambda_{k}=r_{\beta_{k}}\left(\lambda_{k-1}\right)$ and $\lambda_{k-1}\left(\beta_{k}^{\vee}\right)<0$ for $k=1,2, \ldots, s$, where for a positive real root $\beta$, we denote by $r_{\beta}$ the reflection with respect to $\beta$, and by $\beta^{\vee}$ the dual root of $\beta$. Then we define $\operatorname{dist}(\mu, \nu)$ to be the maximal length $s$ among all possible such sequences.

Remark 4. Assume that $\lambda \in P_{+} \cap\left(\mathfrak{h}^{*}\right)^{0}$. It immediately follows that $\mu \geq \nu$ if and only if $\omega^{*}(\mu) \geq \omega^{*}(\nu)$. Moreover, we have $\operatorname{dist}\left(\omega^{*}(\mu), \omega^{*}(\nu)\right)=$ $\operatorname{dist}(\mu, \nu)$ when $\mu \geq \nu$. 
Let $\lambda \in P_{+}, \mu, \nu \in W \lambda$ with $\mu \geq \nu$, and $0<a<1$ a rational number. An $a$-chain for $(\mu, \nu)$ is, by definition, a sequence $\mu=\lambda_{0}>\lambda_{1}>\cdots>\lambda_{r}=\nu$ of elements in $W \lambda$ such that $\operatorname{dist}\left(\lambda_{i}, \lambda_{i-1}\right)=1$ and $\lambda_{i}=r_{\beta_{i}}\left(\lambda_{i-1}\right)$ for some positive real root $\beta_{i}$, and such that $a \lambda_{i-1}\left(\beta_{i}^{\vee}\right) \in \mathbb{Z}$ for all $i=1,2, \ldots, r$.

Here let us consider a pair $\pi=(\underline{\nu} ; \underline{a})$ of a sequence $\underline{\nu}: \nu_{1}>\nu_{2}>\cdots>\nu_{s}$ of elements in $W \lambda$ and a sequence $\underline{a}: 0=a_{0}<a_{1}<\cdots<a_{s}=1$ of rational numbers such that for each $i=1,2, \ldots, s-1$, there exists an $a_{i}$-chain for $\left(\nu_{i}, \nu_{i+1}\right)$. Then we associate to $\pi=(\underline{\nu} ; \underline{a})$ the following path $\pi:[0,1] \rightarrow \mathfrak{h}^{*}$ :

$$
\pi(t)=\sum_{i=1}^{j-1}\left(a_{i}-a_{i-1}\right) \nu_{i}+\left(t-a_{j-1}\right) \nu_{j} \quad \text { for } \quad a_{j-1} \leq t \leq a_{j} .
$$

Such a path is called a Lakshmibai-Seshadri path (L-S path for short) of class $\lambda$. Denote by $\mathbb{B}(\lambda)$ the set of L-S paths of class $\lambda$.

Let us recall the raising and lowering root operators (cf. [Li1]-[Li4]). For convenience, we introduce an extra element $\theta$ that is not a path. For $\pi \in \mathbb{B}(\lambda)$ and $i \in I$, we set

$$
h_{i}^{\pi}(t):=(\pi(t))\left(\alpha_{i}^{\vee}\right), \quad m_{i}^{\pi}:=\min \left\{h_{i}^{\pi}(t) \mid t \in[0,1]\right\} .
$$

First we define the raising root operator $e_{i}$ with respect to the simple root $\alpha_{i}$. We define $e_{i} \theta:=\theta$, and $e_{i} \pi:=\theta$ for $\pi \in \mathbb{B}(\lambda)$ with $m_{i}^{\pi}>-1$. If $m_{i}^{\pi} \leq-1$, then we can take the following points:

$$
\begin{aligned}
& t_{1}:=\min \left\{t \in[0,1] \mid h_{i}^{\pi}(t)=m_{i}^{\pi}\right\}, \\
& t_{0}:=\max \left\{t^{\prime} \in\left[0, t_{1}\right] \mid h_{i}^{\pi}(t) \geq m_{i}^{\pi}+1 \text { for all } t \in\left[0, t^{\prime}\right]\right\} .
\end{aligned}
$$

We set

$$
\left(e_{i} \pi\right)(t):= \begin{cases}\pi(t) & \text { if } 0 \leq t \leq t_{0} \\ \pi(t)-\left(h_{i}^{\pi}(t)-m_{i}^{\pi}-1\right) \alpha_{i} & \text { if } t_{0} \leq t \leq t_{1} \\ \pi(t)+\alpha_{i} & \text { if } t_{1} \leq t \leq 1\end{cases}
$$

The lowering root operator $f_{i}$ is defined in a similar fashion. We define $f_{i} \theta:=\theta$, and $f_{i} \pi:=\theta$ for $\pi \in \mathbb{B}(\lambda)$ with $h_{i}^{\pi}(1)-m_{i}^{\pi}<1$. If $h_{i}^{\pi}(1)-m_{i}^{\pi} \geq 1$, then we can take the following points:

$$
\begin{aligned}
& t_{0}:=\max \left\{t \in[0,1] \mid h_{i}^{\pi}(t)=m_{i}^{\pi}\right\}, \\
& t_{1}:=\min \left\{t^{\prime} \in\left[t_{0}, 1\right] \mid h_{i}^{\pi}(t) \geq m_{i}^{\pi}+1 \text { for all } t \in\left[t^{\prime}, 1\right]\right\} .
\end{aligned}
$$


We set

$$
\left(f_{i} \pi\right)(t):= \begin{cases}\pi(t) & \text { if } 0 \leq t \leq t_{0} \\ \pi(t)-\left(h_{i}^{\pi}(t)-m_{i}^{\pi}\right) \alpha_{i} & \text { if } t_{0} \leq t \leq t_{1} \\ \pi(t)-\alpha_{i} & \text { if } t_{1} \leq t \leq 1\end{cases}
$$

Then we know the following.

Theorem 1.3 ([Li1] and [Li2]). Let $\pi \in \mathbb{B}(\lambda)$. If $e_{i} \pi \neq \theta$ (resp. $f_{i} \pi \neq$ $\theta$ ), then $e_{i} \pi \in \mathbb{B}(\lambda)$ (resp. $f_{i} \pi \in \mathbb{B}(\lambda)$ ). Hence the set $\mathbb{B}(\lambda) \cup\{\theta\}$ is stable under the action of the root operators. Moreover, every element $\pi \in \mathbb{B}(\lambda)$ is of the form $\pi=f_{i_{1}} f_{i_{2}} \cdots f_{i_{k}} \pi_{\lambda}$ for some $i_{1}, i_{2}, \ldots, i_{k} \in I$, where $\pi_{\lambda}:=(\lambda ; 0,1)=t \lambda$ is the only element of $\mathbb{B}(\lambda)$ such that $e_{i} \pi_{\lambda}=\theta$ for all $i \in I$. Furthermore, we have

$$
\sum_{\pi \in \mathbb{B}(\lambda)} e(\pi(1))=\operatorname{ch} L(\lambda), \quad \sum_{\pi \in \mathbb{B}_{w}(\lambda)} e(\pi(1))=\operatorname{ch} L_{w}(\lambda),
$$

where $\mathbb{B}_{w}(\lambda):=\left\{\left(\nu_{1}, \ldots, \nu_{s} ; \underline{a}\right) \in \mathbb{B}(\lambda) \mid \nu_{1} \leq w(\lambda)\right\}$ for each $w \in W$.

It is known from [Kas5] et al. that $\mathbb{B}(\lambda)$ has a natural crystal structure isomorphic to $\mathcal{B}(\lambda)$. Namely, we have the following theorem (see [La2] for the second assertion).

Theorem 1.4. $\quad$ There exists a unique bijection $\Phi: \mathcal{B}(\lambda) \stackrel{\sim}{\rightarrow} \mathbb{B}(\lambda)$ such that

$$
\Phi\left(F_{i_{1}} F_{i_{2}} \cdots F_{i_{k}} \bar{u}_{\lambda}\right)=f_{i_{1}} f_{i_{2}} \cdots f_{i_{k}} \pi_{\lambda} .
$$

Moreover, $\Phi\left(\mathcal{B}_{w}(\lambda)\right)=\mathbb{B}_{w}(\lambda)$ for each $w \in W$.

At the end of this subsection, we recall the main result of [NS1]. Let $\lambda \in P_{+} \cap\left(\mathfrak{h}^{*}\right)^{0}$. For $\pi \in \mathbb{B}(\lambda)$, we define a path $\omega^{*}(\pi):[0,1] \rightarrow \mathfrak{h}^{*}$ by $\left(\omega^{*}(\pi)\right)(t):=\omega^{*}(\pi(t))$. Then we deduce that $\mathbb{B}(\lambda)$ and $\mathbb{B}_{w}(\lambda)$ with $w \in \widetilde{W}$ are $\omega^{*}$-stable (cf. [NS1, Lemma 3.1.1] and Remark 4). Denote by $\mathbb{B}^{0}(\lambda)$ the set of L-S paths that are fixed by $\omega^{*}$, and set $\mathbb{B}_{w}^{0}(\lambda):=\mathbb{B}_{w}(\lambda) \cap \mathbb{B}^{0}(\lambda)$ for each $w \in \widetilde{W}$.

Theorem 1.5 ([NS1, Theorem 3.2.4]). Let $\lambda \in P_{+} \cap\left(\mathfrak{h}^{*}\right)^{0}$ and $w \in \widetilde{W}$. Set $\widehat{\lambda}:=\left(P_{\omega}^{*}\right)^{-1}(\lambda)$ and $\widehat{w}:=\Theta^{-1}(w)$. Then we have

$$
\mathbb{B}^{0}(\lambda)=P_{\omega}^{*}(\widehat{\mathbb{B}}(\widehat{\lambda})), \quad \mathbb{B}_{w}^{0}(\lambda)=P_{\omega}^{*}\left(\widehat{\mathbb{B}}_{\widehat{w}}(\widehat{\lambda})\right),
$$


where we denote by $\widehat{\mathbb{B}}(\widehat{\lambda})$ the set of $\mathrm{L}-\mathrm{S}$ paths of class $\widehat{\lambda}$ for the orbit Lie algebra $\widehat{\mathfrak{g}}$, and set $\widehat{\mathbb{B}}_{\widehat{w}}(\widehat{\lambda}):=\left\{\left(\widehat{\nu}_{1}, \ldots, \widehat{\nu}_{s} ; \underline{a}\right) \in \widehat{\mathbb{B}}(\widehat{\lambda}) \mid \widehat{\nu}_{1} \preceq \widehat{w}(\widehat{\lambda})\right\}$ with $\preceq$ the relative Bruhat order on $\widehat{W} \widehat{\lambda}$. Here, for $\widehat{\pi} \in \widehat{\mathbb{B}}(\widehat{\lambda})$, we define a path $P_{\omega}^{*}(\widehat{\pi}):[0,1] \rightarrow$ $\left(\mathfrak{h}^{*}\right)^{0}$ by $\left(P_{\omega}^{*}(\widehat{\pi})\right)(t):=P_{\omega}^{*}(\widehat{\pi}(t))$.

\section{$\S 2$. Twining Characters and $q$-twining Characters}

\section{§2.1. The twining characters}

From now on, we always assume that $\lambda \in P_{+} \cap\left(\mathfrak{h}^{*}\right)^{0}$ and $w \in \widetilde{W}$. First we consider the linear automorphism $\omega^{-1} \otimes$ id of the Verma module $M(\lambda):=$ $U(\mathfrak{g}) \otimes_{U(\mathfrak{b})} \mathbb{Q}(\lambda)$ of highest weight $\lambda$ over $\mathfrak{g}$, where $\mathbb{Q}(\lambda)$ is the one-dimensional $\mathfrak{b}$-module on which $h \in \mathfrak{h}$ acts by the scalar $\lambda(h)$ and $\mathfrak{n}_{+}$acts trivially. Since this map stabilizes the (unique) maximal proper $\mathfrak{g}$-submodule $N(\lambda)$ of $M(\lambda)$, we obtain the induced $\mathbb{Q}$-linear automorphism $\tau_{\omega}: L(\lambda) \rightarrow L(\lambda)$, where $L(\lambda)=$ $M(\lambda) / N(\lambda)$. It is easily seen that $\tau_{\omega}$ has the following properties:

$$
\tau_{\omega}(x v)=\omega^{-1}(x) \tau_{\omega}(v) \quad \text { for } \quad x \in \mathfrak{g}, v \in L(\lambda)
$$

and $\tau_{\omega}\left(u_{\lambda}\right)=u_{\lambda}$, where $u_{\lambda}$ is a (nonzero) highest weight vector of $L(\lambda)$.

Remark 5. From [N1, Lemma 4.1] (or [NS2, Lemma 2.2.3]), we know that $\tau_{\omega}$ is a unique endomorphism of $L(\lambda)$ with the properties above.

The twining character $\operatorname{ch}^{\omega}(L(\lambda))$ of $L(\lambda)$ is defined to be the formal sum

$$
\operatorname{ch}^{\omega}(L(\lambda)):=\sum_{\chi \in\left(\mathfrak{h}^{*}\right)^{0}} \operatorname{tr}\left(\left.\tau_{\omega}\right|_{L(\lambda)_{\chi}}\right) e(\chi) .
$$

Since $\tau_{\omega}\left(L(\lambda)_{\chi}\right)=L(\lambda)_{\omega^{*}(\chi)}$ for all $\chi \in \mathfrak{h}^{*}$ and $\operatorname{dim} L(\lambda)_{w(\lambda)}=1$ for all $w \in W$, we see that the Demazure module $L_{w}(\lambda)$ is $\tau_{\omega}$-stable for all $w \in \widetilde{W}$. Hence we can define the twining character $\operatorname{ch}^{\omega}\left(L_{w}(\lambda)\right)$ of $L_{w}(\lambda)$ by

$$
\operatorname{ch}^{\omega}\left(L_{w}(\lambda)\right):=\sum_{\chi \in\left(\mathfrak{h}^{*}\right)^{0}} \operatorname{tr}\left(\left.\tau_{\omega}\right|_{L_{w}(\lambda)_{\chi}}\right) e(\chi) .
$$

\section{§2.2. The $q$-twining characters}

In this subsection, we introduce the $q$-twining characters of $V(\lambda)$ and $V_{w}(\lambda)$, which are $q$-analogues of $\operatorname{ch}^{\omega}(L(\lambda))$ and $\operatorname{ch}^{\omega}\left(L_{w}(\lambda)\right)$, respectively (see Proposition 2.1 below). 
By $(1.10)$, we have a $\mathbb{Q}(q)$-linear automorphism $\tau_{\omega_{q}}: V(\lambda) \rightarrow V(\lambda)$ induced from $\omega_{q}^{-1}: U_{q}^{-}(\mathfrak{g}) \rightarrow U_{q}^{-}(\mathfrak{g})$. As in the usual Lie algebra case in Section $2.1, \tau_{\omega_{q}}$ has the following properties:

$$
\tau_{\omega_{q}}(x v)=\omega_{q}^{-1}(x) \tau_{\omega_{q}}(v) \quad \text { for } \quad x \in U_{q}(\mathfrak{g}), v \in V(\lambda)
$$

and $\tau_{\omega_{q}}\left(u_{\lambda}\right)=u_{\lambda}$, where $u_{\lambda}$ is a (nonzero) highest weight vector of $V(\lambda)$.

Remark 6. In a similar way to the proof of [N1, Lemma 4.1], we can show that $\tau_{\omega_{q}}$ is a unique endomorphism of $V(\lambda)$ with the properties above.

The $q$-twining character $\operatorname{ch}_{q}^{\omega}(V(\lambda))$ of $V(\lambda)$ is defined to be the formal sum

$$
\operatorname{ch}_{q}^{\omega}(V(\lambda)):=\sum_{\chi \in\left(\mathfrak{h}^{*}\right)^{0}} \operatorname{tr}\left(\left.\tau_{\omega_{q}}\right|_{V(\lambda)_{\chi}}\right) e(\chi)
$$

We easily see that the quantum Demazure module $V_{w}(\lambda)$ is $\tau_{\omega_{q}}$-stable for every $w \in \widetilde{W}$. Hence we can define the $q$-twining character $\operatorname{ch}_{q}^{\omega}\left(V_{w}(\lambda)\right)$ of $V_{w}(\lambda)$ by

$$
\operatorname{ch}_{q}^{\omega}\left(V_{w}(\lambda)\right):=\sum_{\chi \in\left(\mathfrak{h}^{*}\right)^{0}} \operatorname{tr}\left(\left.\tau_{\omega_{q}}\right|_{V_{w}(\lambda)_{\chi}}\right) e(\chi) .
$$

Remark 7. Naively the traces of $\tau_{\omega_{q}}$ above are elements of $\mathbb{Q}(q)$. In fact, they are elements of $\mathbb{Q}\left[q, q^{-1}\right]$ (see Proposition 2.1 below).

Here let us recall some facts from [Ja, Sections 5.12 through 5.15]. Let $V(\lambda)_{\mathbb{Q}}$ (resp. $\left.V(\lambda)_{\chi, \mathbb{Q}}\right)$ be the $\mathbb{Q}\left[q, q^{-1}\right]$-submodule of $V(\lambda)$ generated by all elements of the form $Y_{i_{1}} Y_{i_{2}} \cdots Y_{i_{k}} u_{\lambda}$ (resp. with $\alpha_{i_{1}}+\alpha_{i_{2}}+\cdots+\alpha_{i_{k}}=\lambda-\chi$ ). It is clear that all $V(\lambda)_{\chi, \mathbb{Q}}$ are finitely generated, torsion free $\mathbb{Q}\left[q, q^{-1}\right]$-modules. Therefore they are free $\mathbb{Q}\left[q, q^{-1}\right]$-modules of finite rank because $\mathbb{Q}\left[q, q^{-1}\right]$ is a principal ideal domain. We also know that the natural map $\mathbb{Q}(q) \otimes_{\mathbb{Q}\left[q, q^{-1}\right]}$ $V(\lambda)_{\mathbb{Q}} \rightarrow V(\lambda)$ (given by $a \otimes v \rightarrow a v$ ) is a $\mathbb{Q}(q)$-linear isomorphism.

Now we consider $\mathbb{Q}$ as a $\mathbb{Q}\left[q, q^{-1}\right]$-module by the evaluation at $q=1$. Set $V:=\mathbb{Q} \otimes_{\mathbb{Q}\left[q, q^{-1}\right]} V(\lambda)_{\mathbb{Q}}$ and $V_{\chi}:=\mathbb{Q} \otimes_{\mathbb{Q}\left[q, q^{-1}\right]} V(\lambda)_{\chi}, \mathbb{Q}$. It follows from [Ja, Lemma 5.12] that $V(\lambda)_{\mathbb{Q}}$ is stable under the actions of $X_{i}, Y_{i}$, and $\left(q^{h}-\right.$ $\left.q^{-h}\right) /\left(q-q^{-1}\right)$ for $i \in I, h \in P^{\vee}$. Thus we obtain endomorphisms $x_{i}, y_{i}$, and $h$ of $V$ defined by

$$
x_{i}:=1 \otimes X_{i}, \quad y_{i}:=1 \otimes Y_{i}, \quad \text { and } \quad h:=1 \otimes\left(q^{h}-q^{-h}\right) /\left(q-q^{-1}\right),
$$

respectively. From [Ja, Lemmas 5.13 and 5.14], we know that the endomorphisms $x_{i}, y_{i}$, and $h$ of $V$ satisfy the Serre relations, and hence that these endomorphisms make $V$ into a $\mathfrak{g}$-module. Moreover, $V \cong L(\lambda)$ as $\mathfrak{g}$-modules, and the image of $V_{\chi}$ by this $\mathfrak{g}$-module isomorphism is $L(\lambda)_{\chi}$ for all $\chi \in \mathfrak{h}^{*}$. Taking these facts into account, we show the following proposition. 
Proposition 2.1. Let $\chi \in\left(\mathfrak{h}^{*}\right)^{0}$ and $w \in \widetilde{W}$. Then $\operatorname{tr}\left(\left.\tau_{\omega_{q}}\right|_{V(\lambda)_{\chi}}\right)$ and $\operatorname{tr}\left(\left.\tau_{\omega_{q}}\right|_{V_{w}(\lambda)_{\chi}}\right)$ are elements of $\mathbb{Q}\left[q, q^{-1}\right]$. Moreover, we have

$$
\left.\operatorname{tr}\left(\left.\tau_{\omega_{q}}\right|_{V(\lambda)_{\chi}}\right)\right|_{q=1}=\operatorname{tr}\left(\left.\tau_{\omega}\right|_{L(\lambda)_{\chi}}\right),\left.\quad \operatorname{tr}\left(\left.\tau_{\omega_{q}}\right|_{V_{w}(\lambda)_{\chi}}\right)\right|_{q=1}=\operatorname{tr}\left(\left.\tau_{\omega}\right|_{L_{w}(\lambda)_{\chi}}\right),
$$

and hence

$$
\left.\operatorname{ch}_{q}^{\omega}(V(\lambda))\right|_{q=1}=\operatorname{ch}^{\omega}(L(\lambda)),\left.\quad \operatorname{ch}_{q}^{\omega}\left(V_{w}(\lambda)\right)\right|_{q=1}=\operatorname{ch}^{\omega}\left(L_{w}(\lambda)\right) .
$$

Proof. It can easily be checked that $V(\lambda)_{\mathbb{Q}}$ is $\tau_{\omega_{q}}$-stable, and the following diagram commutes:

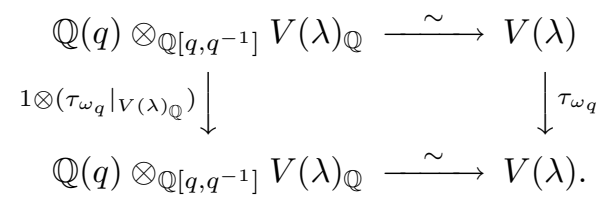

Since $V(\lambda)_{\chi, \mathbb{Q}}$ is a free $\mathbb{Q}\left[q, q^{-1}\right]$-module, we can define the trace of $\left.\tau_{\omega_{q}}\right|_{V(\lambda)_{\chi}, \mathbb{Q}}$ for each $\chi \in\left(\mathfrak{h}^{*}\right)^{0}$. Note that a basis of $V(\lambda)_{\chi, \mathbb{Q}}$ over $\mathbb{Q}\left[q, q^{-1}\right]$ is also a basis of $V(\lambda)_{\chi}$ over $\mathbb{Q}(q)$. We obtain from the commutative diagram above that

$$
\operatorname{tr}\left(\left.\tau_{\omega_{q}}\right|_{V(\lambda)_{\chi}}\right)=\operatorname{tr}\left(\left.\tau_{\omega_{q}}\right|_{V(\lambda)_{\chi, \mathbb{Q}}}\right) \in \mathbb{Q}\left[q, q^{-1}\right] \quad \text { for all } \chi \in\left(\mathfrak{h}^{*}\right)^{0} .
$$

Now let $w \in \widetilde{W}$, and take $u_{w(\lambda)} \in V(\lambda)_{w(\lambda), \mathbb{Q}} \backslash\{0\}$. Here we remark that the rank of the free $\mathbb{Q}\left[q, q^{-1}\right]$-module $V(\lambda)_{w(\lambda), \mathbb{Q}}$ is one. We define $V_{w}(\lambda)_{\mathbb{Q}}$ to be the $\mathbb{Q}\left[q, q^{-1}\right]$-submodule of $V(\lambda)$ generated by the elements of the form $X_{i_{1}} X_{i_{2}} \cdots X_{i_{k}} u_{w(\lambda)}$. It is clear that $V_{w}(\lambda)_{\mathbb{Q}}$ is $\tau_{\omega_{q}}$-stable. Since $V(\lambda)_{\mathbb{Q}}$ is stable under the action of $X_{i}$, we see that $V_{w}(\lambda)_{\mathbb{Q}}$ is a $\mathbb{Q}\left[q, q^{-1}\right]$-submodule of $V(\lambda)_{\mathbb{Q}}$. We set $V_{w}(\lambda)_{\chi, \mathbb{Q}}:=V_{w}(\lambda)_{\mathbb{Q}} \cap V(\lambda)_{\chi, \mathbb{Q}}$. Then we immediately obtain the following commutative diagram:

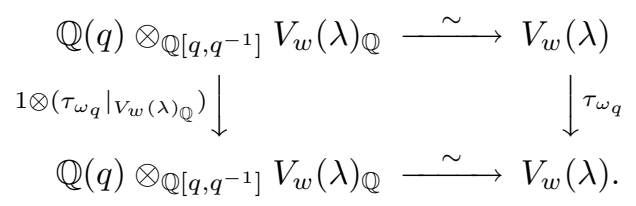

Hence, in the same way as above, we have

$$
\operatorname{tr}\left(\left.\tau_{\omega_{q}}\right|_{V_{w}(\lambda)_{\chi}}\right)=\operatorname{tr}\left(\left.\tau_{\omega_{q}}\right|_{V_{w}(\lambda)_{\chi}, \mathbb{Q}}\right) \in \mathbb{Q}\left[q, q^{-1}\right] \quad \text { for all } \chi \in\left(\mathfrak{h}^{*}\right)^{0},
$$


thereby completing the proof of the first assertion.

Next we show the equalities (2.5). Note that the $\mathbb{Q}$-linear automorphism $\tau_{\omega}^{\prime}:=1 \otimes\left(\left.\tau_{\omega_{q}}\right|_{V(\lambda)_{\mathbb{Q}}}\right)$ of $V:=\mathbb{Q} \otimes_{\mathbb{Q}\left[q, q^{-1}\right]} V(\lambda)_{\mathbb{Q}}$ satisfies $\tau_{\omega}^{\prime}(x v)=\omega^{-1}(x) \tau_{\omega}^{\prime}(v)$ for $x \in \mathfrak{g}, v \in V$, and $\tau_{\omega}^{\prime}\left(1 \otimes u_{\lambda}\right)=1 \otimes u_{\lambda}$. Hence it follows from Remark 5 that the following diagram commutes:

$$
\begin{aligned}
& V=\mathbb{Q} \otimes_{\mathbb{Q}\left[q, q^{-1}\right]} V(\lambda)_{\mathbb{Q}} \stackrel{\sim}{\longrightarrow} L(\lambda) \\
& \tau_{\omega}^{\prime}=1 \otimes\left(\left.\tau_{\omega_{q}}\right|_{V(\lambda) \mathbb{Q}}\right) \downarrow \\
& V=\mathbb{Q} \otimes_{\mathbb{Q}\left[q, q^{-1}\right]} V(\lambda)_{\mathbb{Q}} \stackrel{\sim}{\longrightarrow} L(\lambda) .
\end{aligned}
$$

Remark that, for all $\chi \in\left(\mathfrak{h}^{*}\right)^{0}$,

$$
\operatorname{tr}\left(\left.\tau_{\omega}\right|_{L(\lambda)_{\chi}}\right)=\operatorname{tr}\left(\left.\tau_{\omega}^{\prime}\right|_{V_{\chi}}\right)=1 \otimes_{\mathbb{Q}\left[q, q^{-1}\right]} \operatorname{tr}\left(\left.\tau_{\omega_{q}}\right|_{V(\lambda)_{\chi}, \mathbb{Q}}\right)=\left.\operatorname{tr}\left(\left.\tau_{\omega_{q}}\right|_{V(\lambda)_{\chi}, \mathbb{Q}}\right)\right|_{q=1},
$$

since we regard $\mathbb{Q}$ as a $\mathbb{Q}\left[q, q^{-1}\right]$-module by the evaluation at $q=1$. Combining (2.8) with (2.7), we obtain

$$
\left.\left.\operatorname{tr}\left(\left.\tau_{\omega}\right|_{L(\lambda)_{\chi}}\right) \stackrel{(2.8)}{=} \operatorname{tr}\left(\left.\tau_{\omega_{q}}\right|_{V(\lambda)_{\chi}, \mathbb{Q}}\right)\right|_{q=1} \stackrel{(2.7)}{=} \operatorname{tr}\left(\left.\tau_{\omega_{q}}\right|_{V(\lambda)_{\chi}}\right)\right|_{q=1} \quad \text { for all } \chi \in\left(\mathfrak{h}^{*}\right)^{0},
$$

which proves the first equality of $(2.5)$. By considering $V_{w}:=\mathbb{Q} \otimes_{\mathbb{Q}\left[q, q^{-1}\right]} V_{w}(\lambda)_{\mathbb{Q}}$ for $w \in \widetilde{W}$, we also obtain

$$
\left.\operatorname{tr}\left(\left.\tau_{\omega_{q}}\right|_{V_{w}(\lambda)_{\chi}}\right)\right|_{q=1}=\operatorname{tr}\left(\left.\tau_{\omega}\right|_{L_{w}(\lambda)_{\chi}}\right) \quad \text { for all } \chi \in\left(\mathfrak{h}^{*}\right)^{0}
$$

in the same way. This completes the proof of Proposition 2.1.

\section{$\S 3 . \quad$ Twining Character Formula for Demazure Modules}

The main result of this paper is the following.

Theorem 3.1. Let $\lambda \in P_{+} \cap\left(\mathfrak{h}^{*}\right)^{0}$ and $w \in \widetilde{W}$. Set $\widehat{\lambda}:=\left(P_{\omega}^{*}\right)^{-1}(\lambda)$ and $\widehat{w}:=\Theta^{-1}(w)$. Then we have

$$
\operatorname{ch}^{\omega}\left(L_{w}(\lambda)\right)=P_{\omega}^{*}\left(\operatorname{ch} \widehat{L}_{\widehat{w}}(\widehat{\lambda})\right),
$$

where $\widehat{L}_{\widehat{w}}(\widehat{\lambda})$ is the Demazure module of lowest weight $\widehat{w}(\widehat{\lambda})$ in the irreducible highest weight module $\widehat{L}(\widehat{\lambda})$ of highest weight $\widehat{\lambda}$ over the orbit Lie algebra $\widehat{\mathfrak{g}}$.

We need some lemmas in order to prove this theorem. 
Lemma 3.1. For each $i \in I$, we have $\tau_{\omega_{q}} \circ E_{i}=E_{\omega^{-1}(i)} \circ \tau_{\omega_{q}}$ and $\tau_{\omega_{q}} \circ F_{i}=F_{\omega^{-1}(i)} \circ \tau_{\omega_{q}}$.

Proof. We show only $\tau_{\omega_{q}} \circ E_{i}=E_{\omega^{-1}(i)} \circ \tau_{\omega_{q}}$ since the proof of $\tau_{\omega_{q}} \circ F_{i}=$ $F_{\omega^{-1}(i)} \circ \tau_{\omega_{q}}$ is similar. Let $u=\sum_{k \geq 0} Y_{i}^{(k)} u_{k} \in V(\lambda)$, where $u_{k} \in\left(\operatorname{ker} X_{i}\right) \cap$ $V(\lambda)_{\chi+k \alpha_{i}}$. Since $\omega_{q}^{-1}\left(Y_{i}^{(k)}\right)=Y_{\omega^{-1}(i)}^{(k)}$, we have

$$
\tau_{\omega_{q}} \circ E_{i}(u)=\sum_{k \geq 0} Y_{\omega^{-1}(i)}^{(k-1)} \tau_{\omega_{q}}\left(u_{k}\right)
$$

On the other hand, $\tau_{\omega_{q}}(u)=\sum_{k \geq 0} Y_{\omega^{-1}(i)}^{(k)} \tau_{\omega_{q}}\left(u_{k}\right) \in V(\lambda)_{\omega^{*}(\chi)}$. Here we note that $\tau_{\omega_{q}}\left(u_{k}\right) \in\left(\operatorname{ker} X_{\omega^{-1}(i)}\right) \cap V(\bar{\lambda})_{\omega^{*}(\chi)+k \alpha_{\omega^{-1}(i)}}$. Hence, by the uniqueness of the expression of $\tau_{\omega_{q}}(u)$, we have

$$
E_{\omega^{-1}(i)} \circ \tau_{\omega_{q}}(u)=\sum_{k \geq 0} Y_{\omega^{-1}(i)}^{(k-1)} \tau_{\omega_{q}}\left(u_{k}\right) .
$$

Therefore we obtain $\tau_{\omega_{q}} \circ E_{i}(u)=E_{\omega^{-1}(i)} \circ \tau_{\omega_{q}}(u)$ for all $u \in V(\lambda)$, thereby completing the proof.

This lemma implies that $\mathcal{L}_{0}(\lambda)$ is $\tau_{\omega_{q}}$-stable. Hence we have a $\mathbb{Q}$-linear automorphism $\bar{\tau}_{\omega_{q}}$ of $\mathcal{L}_{0}(\lambda) / q \mathcal{L}_{0}(\lambda)$ induced from $\tau_{\omega_{q}}$. Then, by the definition of $\bar{\tau}_{\omega_{q}}$ and Lemma 3.1 , we can easily check that the set $\mathcal{B}(\lambda)$ is $\bar{\tau}_{\omega_{q}}$-stable. Moreover, by Theorem 1.4, we have the following commutative diagram:

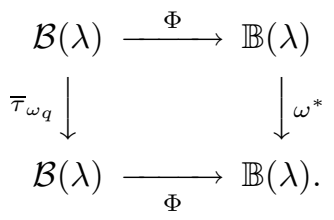

Here we have used the fact that $\omega^{*} \circ e_{i}=e_{\omega^{-1}(i)} \circ \omega^{*}$ and $\omega^{*} \circ f_{i}=f_{\omega^{-1}(i)} \circ \omega^{*}$ ([NS1, Lemma 3.1.1]). The next lemma immediately follows from the commutative diagram (3.2) and Theorem 1.4 , since $\mathbb{B}_{w}(\lambda)$ is $\omega^{*}$-stable for all $w \in \widetilde{W}$.

Lemma 3.2. Let $w \in \widetilde{W}$. Then $\mathcal{B}_{w}(\lambda)$ is stable under $\bar{\tau}_{\omega_{q}}$. Hence we obtain the following commutative diagram:

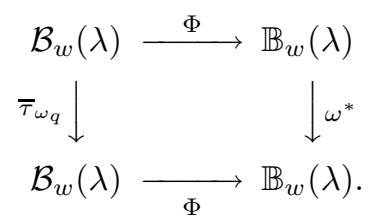


Because $\psi \circ \tau_{\omega_{q}}=\tau_{\omega_{q}} \circ \psi$, we see that $\mathcal{L}_{\infty}(\lambda)$ is also $\tau_{\omega_{q}}$-stable. Since $V_{\mathbb{Q}}(\lambda)$ is obviously $\tau_{\omega_{q}}$-stable, we deduce that $E(\lambda)$ is $\tau_{\omega_{q}}$-stable.

Lemma 3.3. $\tau_{\omega_{q}} \circ G_{\lambda}=G_{\lambda} \circ \bar{\tau}_{\omega_{q}}$.

Proof. Remark that $\left\{G_{\lambda}(b) \mid b \in \mathcal{B}(\lambda)\right\}$ is a basis of the $\mathbb{Q}$-vector space $E(\lambda)$. Hence, for $b \in \mathcal{B}(\lambda)$, we have $\tau_{\omega_{q}}\left(G_{\lambda}(b)\right)=\sum_{b^{\prime} \in \mathcal{B}(\lambda)} c_{b^{\prime}} G_{\lambda}\left(b^{\prime}\right)$ for some $c_{b^{\prime}} \in \mathbb{Q}$ since $E(\lambda)$ is $\tau_{\omega_{q}}$-stable. Then we obtain $\bar{\tau}_{\omega_{q}}(b)=\sum_{b^{\prime} \in \mathcal{B}(\lambda)} c_{b^{\prime}} b^{\prime}$ in $\mathcal{L}_{0}(\lambda) / q \mathcal{L}_{0}(\lambda)$. Put $b^{\prime \prime}:=\bar{\tau}_{\omega_{q}}(b) \in \mathcal{B}(\lambda)$. Because $\mathcal{B}(\lambda)$ is a basis of the $\mathbb{Q}$ vector space $\mathcal{L}_{0}(\lambda) / q \mathcal{L}_{0}(\lambda)$, we see that $c_{b^{\prime \prime}}=1$ and $c_{b^{\prime}}=0$ for all $b^{\prime} \in \mathcal{B}(\lambda)$, $b^{\prime} \neq b^{\prime \prime}$. Hence we obtain $\tau_{\omega_{q}}\left(G_{\lambda}(b)\right)=G_{\lambda}\left(b^{\prime \prime}\right)=G_{\lambda}\left(\bar{\tau}_{\omega_{q}}(b)\right)$, as desired.

Proof of Theorem 3.1. By combining Lemmas 3.2 and 3.3, we see that the set $\left\{G_{\lambda}(b) \mid b \in \mathcal{B}_{w}(\lambda)\right\}$ is $\tau_{\omega_{q}}$-stable. Because $\left\{G_{\lambda}(b) \mid b \in \mathcal{B}_{w}(\lambda) \cap \mathcal{B}(\lambda)_{\chi}\right\}$ is a basis of the $\chi$-weight space $V_{w}(\lambda)_{\chi}$ of $V_{w}(\lambda)$ over $\mathbb{Q}(q)$ (see (1.14)), we obtain

$$
\operatorname{tr}\left(\left.\tau_{\omega_{q}}\right|_{V_{w}(\lambda)_{\chi}}\right)=\#\left\{G_{\lambda}(b) \mid \tau_{\omega_{q}}\left(G_{\lambda}(b)\right)=G_{\lambda}(b), b \in \mathcal{B}_{w}(\lambda) \cap \mathcal{B}(\lambda)_{\chi}\right\}
$$

for $\chi \in\left(\mathfrak{h}^{*}\right)^{0}$ (note that if an endomorphism $f$ on a finite-dimensional vector space $V$ stabilizes a basis of $V$, then the trace of $f$ on $V$ is equal to the number of basis elements fixed by $f$ ). By Lemma 3.3 again, we get

$$
\operatorname{tr}\left(\left.\tau_{\omega_{q}}\right|_{V_{w}(\lambda)_{\chi}}\right)=\#\left\{b \in \mathcal{B}_{w}(\lambda) \cap \mathcal{B}(\lambda)_{\chi} \mid \bar{\tau}_{\omega_{q}}(b)=b\right\}
$$

and hence

$$
\operatorname{ch}_{q}^{\omega}\left(V_{w}(\lambda)\right)=\sum_{b \in \mathcal{B}_{w}^{0}(\lambda)} e(\operatorname{wt}(b))
$$

where $\operatorname{wt}(b):=\chi$ if $b \in \mathcal{B}(\lambda)_{\chi}$, and $\mathcal{B}_{w}^{0}(\lambda)$ is the set of elements of $\mathcal{B}_{w}(\lambda)$ fixed by $\bar{\tau}_{\omega_{q}}$. The commutative diagram (3.3) implies that

$$
\operatorname{ch}_{q}^{\omega}\left(V_{w}(\lambda)\right) \stackrel{(3.4)}{=} \sum_{b \in \mathcal{B}_{w}^{0}(\lambda)} e(\operatorname{wt}(b)) \stackrel{(3.3)}{=} \sum_{\pi \in \mathbb{B}_{w}^{0}(\lambda)} e(\pi(1)) .
$$

We see from Theorems 1.3 and 1.5 that the right-hand side of the above equality coincides with $P_{\omega}^{*}\left(\operatorname{ch} \widehat{L}_{\widehat{w}}(\widehat{\lambda})\right)$, where $\widehat{\lambda}:=\left(P_{\omega}^{*}\right)^{-1}(\lambda)$ and $\widehat{w}:=\Theta^{-1}(w)$. Therefore we obtain

$$
\operatorname{ch}_{q}^{\omega}\left(V_{w}(\lambda)\right)=P_{\omega}^{*}\left(\operatorname{ch} \widehat{L}_{\widehat{w}}(\widehat{\lambda})\right) .
$$


Since the right-hand side is independent of $q$, we find that $\left.\operatorname{ch}_{q}^{\omega}\left(V_{w}(\lambda)\right)\right|_{q=1}=$ $P_{\omega}^{*}\left(\operatorname{ch} \widehat{L}_{\widehat{w}}(\widehat{\lambda})\right)$. Combining this with (2.6), we finally arrive at the conclusion that

$$
\operatorname{ch}^{\omega}\left(L_{w}(\lambda)\right)=P_{\omega}^{*}\left(\operatorname{ch} \widehat{L}_{\widehat{w}}(\widehat{\lambda})\right) .
$$

Thus we have proved Theorem 3.1.

Remark 8. By replacing $V_{w}(\lambda)$ by $V(\lambda)$ and $L_{w}(\lambda)$ by $L(\lambda)$ in the arguments above, we can give another proof of the twining character formula for the integrable highest weight module $L(\lambda)$, which is the main result of [FSS] ([FRS $]$ ).

\section{Acknowledgements}

I express my sincere thanks to Professor Satoshi Naito, who lead me to the theories of path models and twining characters. This paper could not be written without his guidance.

\section{References}

[D] Demazure, M., Une nouvelle formule des caractères, Bull. Sci. Math., 98 (1974), 163-172.

[FRS] Fuchs, J., Ray, U. and Schweigert, C., Some automorphisms of generalized Kac-Moody algebras, J. Algebra, 191 (1997), 518-540.

[FSS] Fuchs, J., Schellekens, B. and Schweigert, C., From Dynkin diagram symmetries to fixed point structures, Comm. Math. Phys., 180 (1996), 39-97.

[Ja] Jantzen, J. C., Lectures on Quantum Groups, Grad. Stud. Math., 6, Amer. Math. Soc., Providence, 1996.

[Jo] Joseph, A., Quantum Groups and Their Primitive Ideals, Ergeb. Math. Grenzgeb., 29, Springer-Verlag, Berlin, 1995.

[Kac] Kac, V. G., Infinite Dimensional Lie Algebras, 3rd Edition, Cambridge University Press, Cambridge, 1990.

[KN] Kaneda, M. and Naito, S., A twining character formula for Demazure modules, to appear in Transform. Groups.

[KK] Kang, S.-J. and Kwon, J.-H., Graded Lie superalgebras, supertrace formula, and orbit Lie superalgebras, Proc. London Math. Soc., 81 (2000), 675-724.

[Kas1] Kashiwara, M., On crystal bases of the $q$-analogue of universal enveloping algebras, Duke Math. J., 63 (1991), 465-516.

[Kas2] - Global crystal bases of quantum groups, Duke Math. J., 69 (1993), 455-485.

[Kas3] , The crystal base and Littelmann's refined Demazure character formula, Duke Math. J., 71 (1993), 839-858.

[Kas4] - On crystal bases, Representations of Groups (Banff, 1994), CMS Conf. Proc., 16, 155-197, Amer. Math. Soc., Providence, 1995.

[Kas5] - Similarity of crystal bases, Lie Algebras and Their Representations (Seoul, 1995), Contemp. Math., 194, 177-186, Amer. Math. Soc., Providence, 1996.

[Ku] Kumar, S., Demazure character formula in arbitrary Kac-Moody setting, Invent. Math., 89 (1987), 395-423. 
[La1] Lakshmibai, V., Bases for quantum Demazure modules II, Algebraic groups and their generalizations: quantum and infinite-dimensional methods (University Park, 1991), Proc. Sympos. Pure Math., 56(2), 149-168, Amer. Math. Soc., Providence, 1994.

[La2] - Bases for quantum Demazure modules, Representations of Groups (Banff, 1994), CMS Conf. Proc., 16, 199-216, Amer. Math. Soc., Providence, 1995.

[Li1] Littelmann, P., A Littlewood-Richardson rule for symmetrizable Kac-Moody algebras, Invent. Math., 116 (1994), 329-346.

[Li2] - Paths and root operators in representation theory, Ann. of Math., 142 (1995), 499-525.

[Li3] - Characters of representations and paths in $\mathfrak{h}_{\mathbb{R}}^{*}$, Representation Theory and Automorphic Forms (Edinburgh, 1996), Proc. Sympos. Pure Math., 61, 29-49, Amer. Math. Soc., Providence, 1997.

[Li4] - The path model, the quantum Frobenius map and standard monomial theory, Algebraic Groups and their Representations (Cambridge, 1997), NATO Adv. Sci. Inst. Ser. C, 517, 175-212, Acad. Publ., Dordrecht, 1998.

[Lu] Lusztig, G., Introduction to Quantum Groups, Progr. Math., 110, Birkhäuser, Boston, 1993.

[M] Mathieu, O., Formules de caractères pour les algèbres de Kac-Moody générales, Astérisque, 159-160 (1988).

[MP] Moody, R. V. and Pianzola, A., Lie Algebras with Triangular Decompositions, Canadian Mathematical Society Series of Monographs and Advanced Texts, WileyInterscience, New York, 1995.

[N1] Naito, S., Twining character formula of Kac-Wakimoto type for affine Lie algebras, Preprint.

[N2] - Twining characters and Kostant's homology formula, to appear in Tohoku Math. J.

[N3] - Twining characters, Kostant's homology formula, and the Bernstein-GelfandGelfand resolution, J. Math. Kyoto Univ., 42 (2002), 83-103.

[N4] - Twining character formula of Borel-Weil-Bott type, to appear in J. Math. Sci. Univ. Tokyo.

[NS1] Naito, S. and Sagaki, D., Lakshmibai-Seshadri paths fixed by a diagram automorphism, J. Algebra, 245 (2001), 395-412.

[NS2] Certain modules with twining maps and decomposition rules of Littelmann type, Comm. Algebra, 30 (2002).

Note added in proof: In this paper, we imposed the linking condition (1.6) on the diagram automorphism $\omega: I \rightarrow I$. However, this condition is not essential, since Theorem 1.5 still holds without it. For details, see our paper: Naito, S. and Sagaki, D., Standard paths and standard monomials fixed by a diagram automorphism, $J$. Algera, 251 (2002). 